\title{
Entanglement and the speed of evolution of multi-partite quantum systems
}

\author{
C Zander ${ }^{1}$, A R Plastino ${ }^{1,2}$, A Plastino ${ }^{2}$ and M Casas ${ }^{3}$ \\ ${ }^{1}$ Physics Department, University of Pretoria, Pretoria 0002, South Africa \\ 2 National University La Plata-CONICET, C.C. 727, 1900 La Plata, Argentina \\ ${ }^{3}$ Department de Física and IMEDEA-CSIC, Universitat de les Illes Balears, 07122 Palma de \\ Mallorca, Spain \\ E-mail: arplastino@maple.up.ac.za
}

Received 24 July 2006, in final form 4 December 2006

Published 28 February 2007

Online at stacks.iop.org/JPhysA/40/2861

\begin{abstract}
There exists an interesting relationship between entanglement and the time evolution of composite quantum systems: quantum entanglement enhances the 'speed' of evolution of certain quantum states, as measured by the time needed to reach an orthogonal state. Previous research done on this subject has been focused upon comparing extreme cases (highly entangled states versus separable states) or upon bi-partite systems. In the present contribution we explore the aforementioned connection (between entanglement and time evolution) in the cases of two-qubits and $N$-qubits systems, taking into account states of intermediate entanglement. In particular, we investigate a family of energetically symmetric states of low entanglement that saturate the quantum speed bound. We show that, as the number of qubits increases, very little entanglement is needed to reach the quantum speed limit.
\end{abstract}

PACS numbers: 03.65.Ud, 03.67.-a

\section{Introduction}

Quantum entanglement [1] is widely regarded as one of (if not the) most fundamental feature of the quantum picture of the physical world [2-4]. It has been the subject of intense research activity in recent years [5-21]. Quantum entanglement constitutes a physical resource that lies at the basis of important quantum information processes [4] such as quantum teleportation [5], superdense coding [6], and quantum computation [7]. The experimental implementation of these processes may have important practical applications, not only in the communication and computational technologies, but also in other areas, such as quantum metrology [8]. Besides its technological relevance, current research in quantum entanglement is shedding new light upon fundamental aspects of quantum physics, such as, for instance, the emergence of thermodynamic behaviour within composite quantum systems [10]. Another example 
is provided by an interesting relationship between entanglement and the time evolution of composite quantum systems that has recently been established [11-14]: quantum entanglement enhances the 'speed' of evolution of certain quantum states, as measured by the time needed to reach an orthogonal state. The problem of the 'speed' of quantum evolution has been the focus of considerable interest recently, because of its relevance in connection with the physical limits imposed by the basic laws of quantum mechanics on the speed of information processing and information transmission [31-34]. When processing information it is sensible to expect the output state of the computer device to be reasonably distinct from the input state [31]. In quantum mechanics two states are distinguishable if they are orthogonal. Basic computational steps, thus, involve moving from one quantum state to an orthogonal one. Consequently, lower bounds on the time needed to reach an orthogonal state also provide estimations on how fast one can perform elementary computation operations [33]. These, in turn, can be used to estimate fundamental limits on how fast can a physical computer run $[31,33]$. This approach has even been extrapolated to estimate the total number of basic logical operations that has been performed by the universe (regarded as a computing device) during its complete history [34]. The problem of the minimum time necessary to reach an orthogonal state is also relevant in connection with the time-energy Heisenberg uncertainty principle [33], which can be formulated in terms of the minimum time that a system with a given spread in energy requires to evolve to a distinguishable state (that is, to an orthogonal state).

Due to its great importance, both from the fundamental and from the practical points of view, it is imperative to investigate and survey in detail all the implications of the concept of entanglement. The aim of the present contribution is to explore some aspects of the relationship between quantum entanglement and the speed of quantum evolution. In particular, we introduce and investigate a family of energetically symmetric states with low entanglement that saturate the quantum speed limit. To the best of our knowledge, these states are the first symmetric $N$-qubit states saturating the speed bound, besides the $|\mathrm{GHZ}\rangle$ states, that have been reported in the literature.

The paper is organized as follows. In section 2 the bounds on the speed of quantum evolution are briefly reviewed. The connection between entanglement and speed of evolution in two-qubits systems is revisited in section 3, paying special attention to states of low entanglement saturating the quantum speed limit. Some of the results obtained for two qubits are extended to the case of $N$-qubits in section 4. Finally, some conclusions are drawn in section 5 .

\section{Speed of evolution}

A natural measure for the 'speed' of quantum evolution is provided by the time interval $\tau$ that a given initial state $\left|\psi\left(t_{0}\right)\right\rangle$ takes to evolve into an orthogonal state [11, 31],

$$
\left\langle\psi\left(t_{0}\right) \mid \psi\left(t_{0}+\tau\right)\right\rangle=0
$$

Let $E$ denote the energy's expectation value,

$$
E=\langle H\rangle
$$

and $\Delta E$, the energy's uncertainty,

$$
\Delta E=\sqrt{\left\langle H^{2}\right\rangle-\langle H\rangle^{2}} \text {. }
$$

A lower limit for the evolution time $\tau$ to an orthogonal state is given by [11]

$$
\tau_{\min }=\max \left(\frac{\pi \hbar}{2 E}, \frac{\pi \hbar}{2 \Delta E}\right) .
$$




\section{Bi-partite systems}

First we are going to consider a composite system consisting of two qubits. That is, two identical (but distinguishable) subsystems each one described by a two-dimensional Hilbert space. The Hamiltonian governing the evolution of our system is of the form

$$
H=H_{1}+H_{2} \text {, }
$$

where $H_{i}$ is a Hamiltonian acting only on the $i$-qubit. The time evolution operator associated with the Hamiltonian (5) is local and, consequently, the amount of entanglement of the system does not change in time. The two single qubit Hamiltonians $H_{i}$ have the same structure, with eigenstates, $\{|0\rangle,|1\rangle\}$, and corresponding eigenenergies $E_{0}=0, E_{1}=\epsilon$. Hamiltonians like (5) are relevant for the study of some fundamental aspects of quantum entanglement (see for instance [19]) and, particularly, in connection with the problem of the speed of quantum evolution of entangled states (see [11]). Our composite system can be described in terms of the basis $\{|00\rangle,|01\rangle,|10\rangle,|11\rangle\}$, which can be rewritten as $\{|0\rangle,|1\rangle,|2\rangle,|3\rangle\}$. The general state of our two qubit system is then

$$
\left|\psi\left(t_{0}\right)\right\rangle=\sum_{j=0}^{3} a_{j}|j\rangle,
$$

where $a_{j}$ 's are complex coefficients satisfying the normalization requirement,

$$
\sum_{j=0}^{3}\left|a_{j}\right|^{2}=1 .
$$

The degree of mixedness of the marginal density matrix associated with one of the subsystems,

$$
\rho_{1}=\operatorname{Tr}_{2}(|\psi\rangle\langle\psi|),
$$

provides a quantitative characterization of the amount of entanglement of a pure state $|\psi\rangle$ of a bipartite system. This degree of mixedness can be measured in several ways. One possibility is to use the von Neumann entropy $S=-\operatorname{Tr} \rho_{1} \ln \rho_{1}$, leading to an entanglement measure called entropy of entanglement. Another possibility, frequently used in the literature because of its advantages for numerical and analytical computations, is given by the linear entropy, $1-\operatorname{Tr} \rho_{1}^{2}[16-18]$. This choice leads to the entanglement measure

$$
\mathcal{E}(|\psi\rangle)=2\left[1-\operatorname{Tr}\left(\rho_{1}^{2}\right)\right]
$$

which is the one we are going to use in the present work. In terms of the measure $\mathcal{E}(|\psi\rangle)$, factorizable pure states (which have zero entanglement) are characterized by $\mathcal{E}\left(\left|\phi_{1}\right\rangle\left|\phi_{2}\right\rangle\right)=0$. On the other hand, states of maximum entanglement, such as

$$
\left|\psi_{E P R}\right\rangle=\frac{1}{\sqrt{2}}(|00\rangle+|11\rangle)
$$

have $\mathcal{E}\left(\left|\psi_{E P R}\right\rangle\right)=1$. Intermediate degrees of entanglement correspond to values $0<$ $\mathcal{E}(|\psi\rangle)<1$. It must be mentioned that the main results that are going to be reported here do not depend on the particular measure (9) adopted. Similar results would be obtained if other measures (such as the concurrence or the entropy of entanglement) were used.

In order to characterize those initial states $\left|\psi\left(t_{0}\right)\right\rangle$ that evolve into orthogonal ones, one has to consider the equation

$$
P(x)=\left\langle\psi\left(t_{0}\right) \mid \psi\left(t_{0}+\tau\right)\right\rangle=\left|a_{3}\right|^{2} x^{2}+\left(\left|a_{1}\right|^{2}+\left|a_{2}\right|^{2}\right) x+\left|a_{0}\right|^{2}=0,
$$

where

$$
x=\exp (-\mathrm{i} \epsilon \tau / \hbar)
$$


The initial state (6) evolves to an orthogonal state if and only if the quadratic equation (11) admits at least one root with modulus equal to 1 . This may happen in two different ways: equation (11) may have two complex conjugate roots of modulus 1 , or it may have two real roots, of which one must have modulus equal to 1 . Due to the notation that we are going to introduce shortly, these two cases are going to be designated, respectively, the $\beta$-case and the $s$-case.

\section{1. $\beta$-case}

This is the case where equation (11) has two complex conjugate roots

$$
x=\mathrm{e}^{ \pm \mathrm{i} \beta},
$$

with

$$
\beta=\frac{\epsilon \tau}{\hbar} .
$$

The coefficients appearing in (11) can be written in terms of $\beta$,

$$
\left|a_{0}\right|^{2}=\left|a_{3}\right|^{2}=\frac{1}{2(1-\cos \beta)}, \quad\left|a_{1}\right|^{2}+\left|a_{2}\right|^{2}=-\frac{\cos \beta}{1-\cos \beta}=C .
$$

Expressing the energy's expectation value $\langle E\rangle$ and uncertainty $\Delta E$ in terms of $\beta$ (where $\pi / 2 \leqslant \beta \leqslant \pi)$ it can be verified that

$$
\Delta E \leqslant E,
$$

and consequently,

$$
\frac{\tau}{\tau_{\min }}=\frac{\pi \hbar}{2 \Delta E}=\frac{2 \beta}{\pi \sqrt{1-\cos \beta}} .
$$

In order to get expressions for $\left|a_{1}\right|^{2}$ and $\left|a_{2}\right|^{2}$, one has to introduce a parameter, $0 \leqslant \delta \leqslant 1$ such that

$$
\left|a_{1}\right|^{2}=\delta C, \quad\left|a_{2}\right|^{2}=(1-\delta) C .
$$

Since a global phase factor does not affect the physical properties of a state, one can choose the global phase factor such that $a_{0}$ is real. Introducing three phase parameters

$$
0 \leqslant \gamma_{1}, \gamma_{2}, \gamma_{3} \leqslant 2 \pi
$$

the coefficients $a_{0}, a_{1}, a_{2}$ and $a_{3}$ can be parameterized as follows:

$$
\begin{aligned}
a_{0} & =\frac{1}{\sqrt{2(1-\cos \beta)}}, & a_{1} & =\mathrm{e}^{\mathrm{i} \gamma_{1}} \sqrt{\delta \frac{(-\cos \beta)}{1-\cos \beta},} \\
a_{2} & =\mathrm{e}^{\mathrm{i} \gamma_{2}} \sqrt{(1-\delta) \frac{(-\cos \beta)}{1-\cos \beta}}, & a_{3} & =\mathrm{e}^{\mathrm{i} \gamma_{3}} \frac{1}{\sqrt{2(1-\cos \beta)}} .
\end{aligned}
$$

Consequently, the entanglement $\mathcal{E}\left(\left|\psi\left(t_{0}\right)\right\rangle\right)$ is a function of $\beta$ and of four independent parameters, whereas $\tau / \tau_{\min }$ is only a function of $\beta$ (remember that the entanglement of the system does not change in time). Since we are interested in the relation between entanglement and the speed of time evolution, we have to obtain an analytic expression for the boundary curve of this relation, that is, one has to find the set of parameters yielding the minimum possible entanglement for a given value of $\tau / \tau_{\min }$. This means finding the set of parameters minimizing the value of $\mathcal{E}=2\left[1-\operatorname{Tr}\left(\rho_{1}^{2}\right)\right]$ for each value of $\beta$ in the interval $\pi / 2 \leqslant \beta \leqslant \pi$. For a quantum state characterized by the coefficients (20) we have,

$\mathcal{E}=\frac{1}{(1-\cos \beta)^{2}}\left\{1+4 \sqrt{\delta(1-\delta)} \cos \left(\gamma_{3}-\gamma_{1}-\gamma_{2}\right) \cos \beta+4 \delta(1-\delta) \cos ^{2} \beta\right\}$. 


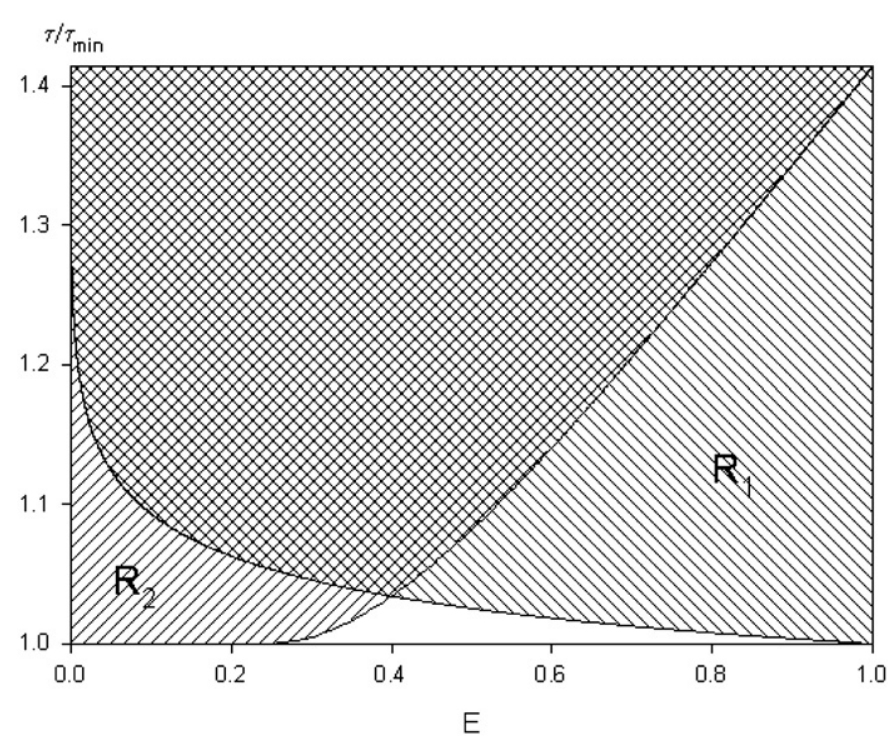

Figure 1. $\mathcal{E}$ versus $\tau / \tau_{\min }$ for the 2-qubit case: the curves bounding regions $R_{1}$ and $R_{2}$ correspond to $\left\{\mathcal{E}(\beta, \delta=1 / 2), \tau / \tau_{\min }(\beta)\right\}$ and $\left\{\mathcal{E}(s, \lambda=1 / 2), \tau / \tau_{\min }(s)\right\}$, respectively.

We see that, for any given value of $\delta$ the minimum value of expression (21) is obtained when

$$
\cos \left(\gamma_{3}-\gamma_{1}-\gamma_{2}\right)=1 \text {. }
$$

In particular, by setting $\gamma_{1}=\gamma_{2}=\gamma_{3}=0$, hence by making all the coefficients real, the measure $\mathcal{E}$ is minimized. Taking the partial derivative of $\mathcal{E}(\beta, \delta)$ with respect to $\delta$ and then choosing $\delta=1 / 2$ gives zero for all $\beta$ in the domain and hence $\delta=1 / 2$ corresponds to a critical point. The second derivative test shows that $\mathcal{E}(\beta, \delta)$ is a convex function of $\delta$ for all allowed $\beta$ and thus the critical point is indeed the global minimum.

From this point on we shall assume the above values for the four parameters. The concomitant expression for the entanglement measure is then given by

$$
\mathcal{E}(\beta)=2-\frac{1-6 \cos \beta+\cos ^{2} \beta}{(-1+\cos \beta)^{2}} .
$$

The parametric plot of $\tau / \tau_{\min }(\beta)$ versus $\mathcal{E}(\beta, \delta=1 / 2)$, with $\beta \in[\pi / 2, \pi]$, yields the curve bounding region $R_{1}$ from below in figure 1 .

Summing up, the $\beta$-case corresponds to the shaded region $R_{1}$ in the $\left(\mathcal{E}, \tau / \tau_{\min }\right)$-plane. All the points in this region are physically realizable.

\section{2. s-case}

In this case equation (11) has two real roots. One of these roots must have modulus equal to 1 , meaning it must be \pm 1 and thus either $\tau=0$ which is impossible since a state can never be orthogonal to itself or

$$
\tau=\frac{\pi \hbar}{\epsilon} \text {. }
$$

Therefore, one root must be -1 . Denoting the other root $s$, the polynomial appearing in equation (11) can be written under the guise

$$
P(x)=\left|a_{3}\right|^{2}\left(x^{2}+(1-s) x-s\right) .
$$


Note that the particular instance of the $\beta$-case corresponding to $\beta=\pi$ coincides with the $s$-case with $s=-1$. The coefficients of $P(x)$ have to be positive, resulting in the restriction $s \leqslant 0$. Comparing expressions (11) and (25) for $P(x)$, and taking into account the normalization requirement $(7)$, results in

$$
\begin{aligned}
& \left|a_{3}\right|^{2}=\frac{1}{2(1-s)}, \\
& \left|a_{0}\right|^{2}=-s\left|a_{3}\right|^{2}=\frac{-s}{2(1-s)}, \\
& \left|a_{1}\right|^{2}+\left|a_{2}\right|^{2}=\frac{1-s}{2(1-s)}=\frac{1}{2} .
\end{aligned}
$$

Introducing four parameters as in the $\beta$-case, we get

$$
\begin{aligned}
a_{0} & =\sqrt{\frac{-s}{2(1-s)}}, & a_{1} & =\mathrm{e}^{\mathrm{i} \mu_{1}} \sqrt{\frac{\lambda}{2}}, \\
a_{2} & =\mathrm{e}^{\mathrm{i} \mu_{2}} \sqrt{\frac{1-\lambda}{2}}, & a_{3} & =\mathrm{e}^{\mathrm{i} \mu_{3}} \frac{1}{\sqrt{2(1-s)}} .
\end{aligned}
$$

Since $\tau$ has the constant value (24), all the states in the $s$-family take the same amount of time (actually maximum possible time) to evolve to an orthogonal state, but what can be different among the states is the dispersion of the energy and hence $\tau_{\min }$. Thus

$$
\frac{\tau}{\tau_{\min }}(s)=\sqrt{\frac{1-6 s+s^{2}}{(-1+s)^{2}}}
$$

changes from state to state.

Once again, we are interested in the boundary curve of the entanglement and speed of time evolution relation. In this case we are looking for the curve in the $\left(\mathcal{E}, \tau / \tau_{\min }\right)$-plane corresponding to the maximum value of $\mathcal{E}$ (maximum entanglement) for any given value of $\tau / \tau_{\min }$. The $\mathcal{E}$-measure associated with a state given by (27) is

$$
\mathcal{E}=\frac{\lambda(1-\lambda)(1-s)^{2}-s}{(1-s)^{2}}-\frac{2}{1-s} \sqrt{-s \lambda(1-\lambda)} \cos \left(\mu_{3}-\mu_{2}-\mu_{1}\right)
$$

For given values of $s$ and $\lambda$, the maximum of $\mathcal{E}$ is achieved when

$$
\cos \left(\mu_{3}-\mu_{2}-\mu_{1}\right)=-1 \text {. }
$$

In particular, one can choose $\mu_{1}=\mu_{2}=0, \mu_{3}=\pi$, and thus make all the coefficients real. Assuming that relation (30) holds, $\mathcal{E}$ becomes a function solely of $s$ and $\lambda$ and, for any given value of $s$, the maximum of $\mathcal{E}$ corresponds to $\lambda=1 / 2$. On the other hand, for given values of $s$ and $\lambda$, the minimum of (29) corresponds to $\cos \left(\mu_{3}-\mu_{2}-\mu_{1}\right)=1$, and letting $\lambda(1-\lambda)=-s /(1-s)^{2}$ yields $\mathcal{E}=0$ (that is, a separable state). In other words, the minimum entanglement compatible with a given value of $s$ (or a given value of $\tau / \tau_{\min }$ ) is zero.

Both $\mathcal{E}$ and $\tau / \tau_{\min }$ (assuming (30)) have the property that for any $u<0$,

$$
\mathcal{E}(s=u, \lambda)=\mathcal{E}\left(s=\frac{1}{u}, \lambda\right), \quad \frac{\tau}{\tau_{\min }}(s=u)=\frac{\tau}{\tau_{\min }}\left(s=\frac{1}{u}\right) .
$$

Thus the case $-1 \leqslant s \leqslant 0$ is equivalent to the case $s \leqslant-1$, and so we are only going to consider $-1 \leqslant s \leqslant 0$. Another property of $\mathcal{E}$ is that for any $0 \leqslant c \leqslant 1$,

$$
\mathcal{E}(s, \lambda=c)=\mathcal{E}(s, \lambda=1-c) .
$$

In order to obtain the family of states that saturate the speed bound, we set $\tau / \tau_{\min }=1 \mathrm{in}$ equation (28). The only solution is $s=0$. The parameter $\lambda$ may adopt any value in the range 
$[0,1]$, with $\lambda=1 / 2$ and $\lambda=0,1$ corresponding, respectively, to the maximum and minimum value of $\mathcal{E}$, namely $1 / 4$ and 0 . In the latter instance the two qubits are not entangled (the state is factorizable). One of the two qubits is in an eigenstate of its Hamiltonian and thus does not evolve in time, nor does it contribute to the dispersion of the energy. The other qubit has maximal dispersion and hence evolves to an orthogonal state at the speed limit. On the other hand, all the states

$$
|\psi(\lambda)\rangle=\sqrt{\frac{\lambda}{2}}|01\rangle+\sqrt{\frac{1-\lambda}{2}}|10\rangle-\frac{1}{\sqrt{2}}|11\rangle
$$

with $0<\lambda<1$, thus with $0<\mathcal{E} \leqslant 1 / 4$, are entangled. We see that, unlike the $\beta$-case (see equation (17)) where only $\beta=\pi / 2$, hence only maximally entangled states saturate the bound, there is now a continuous family of partially entangled states (parameterized by the parameter $\lambda$ ) that saturate the speed bound and evolve as fast as possible. On the opposite side of the quantum speed range, the slowest evolution occurs when $\tau / \tau_{\min }=\sqrt{2}$, thus when $s=-1$. In that case $\lambda=1 / 2$ (together with condition (30)) corresponds to the state

$$
\frac{1}{2}[|0\rangle \otimes(|0\rangle+|1\rangle)+|1\rangle \otimes(|0\rangle-|1\rangle)],
$$

which is clearly maximally entangled (and, consequently, has $\mathcal{E}=1$ ). The set of parameter values $s=-1, \lambda=1 / 2$, together with $\mu_{3}=\mu_{2}=\mu_{1}=0$, corresponds to the state

$$
\frac{1}{2}[|0\rangle|0\rangle+|0\rangle|1\rangle+|1\rangle|0\rangle+|1\rangle|1\rangle]
$$

which also evolves in the slowest way, but has zero entanglement. We thus see that the states of the $s$-family with $\tau / \tau_{\min }=\sqrt{2}$ cover the complete range of amounts of entanglement: from separability to maximum entanglement.

A summary of the above results is provided in figure 1. The parametric plot $\left\{\mathcal{E}(s, \lambda=1 / 2), \tau / \tau_{\min }(s)\right\}$ (taking into account (30)) corresponds to the boundary of the region $R_{2}$. This curve is given (in terms of the parameter $s$ ) by the equations

$$
\begin{aligned}
& \mathcal{E}(s, \lambda=1 / 2)=2-\frac{1}{4}\left[-4 \frac{\sqrt{-s}}{1-s}+\frac{7+s(-10+7 s)}{(-1+s)^{2}}\right], \\
& \frac{\tau}{\tau_{\min }}(s)=\sqrt{\frac{1-6 s+s^{2}}{(-1+s)^{2}}} .
\end{aligned}
$$

Therefore, the $s$-case corresponds to the shaded region $R_{2}$ in the $\left(\mathcal{E}, \frac{\tau}{\tau_{\min }}\right)$-plane. All the points within this region are associated with physically realizable states.

We see that, in a sense, the $\beta$-family (region $R_{1}$ ) and the $s$-family (region $R_{2}$ ) behave in opposite ways. In region $R_{1}$ states evolving faster (that is, with smaller values of $\tau / \tau_{\min }$ ) tend to exhibit increasing entanglement. In contrast, in region $R_{2}$ states with decreasing values of $\tau / \tau_{\min }$ tend to have lower entanglement. However, it must be kept in mind that regions $R_{1}$ and $R_{2}$ also differ in connection with the absolute time $\tau$ needed to reach an orthogonal state. In region $R_{2}$ all states take the same time $\tau$ to evolve into an orthogonal state. In region $R_{1}$, the absolute evolution time to an orthogonal state is minimized by states of maximum entanglement.

The lower boundaries of region $R_{1}$ and region $R_{2}$ intersect at a point corresponding to the parameter values $\beta=1.79841$ and $s=-0.0179989$. Thus the blank region, which corresponds to points in the $\left(\mathcal{E}, \frac{\tau}{\tau_{\min }}\right)$-plane that are not physically allowed, is bounded by $\left\{\mathcal{E}(s, \lambda=1 / 2), \tau / \tau_{\min }(s)\right\}$ for $-0.0179989 \leqslant s \leqslant 0$ and $\left\{\mathcal{E}(\beta, \delta=1 / 2), \tau / \tau_{\min }(\beta)\right\}$ for $\pi / 2 \leqslant \beta \leqslant 1.79841$. These results are consistent with previous research, in the sense that either entanglement or the asymmetry of the state can enhance time evolution. 
The states evolving to an orthogonal state at the highest possible speed (in the sense of the speed bound) have either maximum entanglement $(\mathcal{E}=1)$ or (relatively) low entanglement $(0 \leqslant \mathcal{E} \leqslant 1 / 4)$. Consequently, there is an entanglement gap, given by

$$
\frac{1}{4}<\mathcal{E}<1
$$

corresponding to entanglement values that are not physically permitted for states evolving at the quantum speed limit. Those states saturating the speed bound and having the particular amount of entanglement given by

$$
\mathcal{E}_{\text {ESSLE }}=\frac{1}{4}
$$

are especially interesting because they define a family of energetically symmetric states with low entanglement (ESSLE) that saturate the speed bound. By 'energetically symmetric' states we mean states where the energy's expectation value $E$, and the energy's variance $(\Delta E)^{2}$ are evenly shared among all the subsystems (in this case, the two qubits involved).

\section{4. $N$-qubits ESSLE states saturating the quantum speed limit}

Now we are going to consider a system consisting of $N$ qubits evolving according to the Hamiltonian

$$
H=\sum_{i=1}^{N} H_{i},
$$

where each of the single qubit Hamiltonians $H_{i}$ have eigenstates $|0\rangle,|1\rangle$ with eigenvalues $0, \epsilon$ respectively.

When considering entangled $N$-qubits states, we can take as a reference the GHZ state,

$$
|\mathrm{GHZ}\rangle=\frac{1}{\sqrt{2}}\{|0 \ldots 0\rangle+|1 \ldots 1\rangle\} .
$$

This is an entangled, energetically symmetric state that evolves into an orthogonal state and saturates the quantum speed bound. The time needed for the GHZ state to reach an orthogonal state is

$$
\tau_{\mathrm{GHZ}}=\frac{\pi \hbar}{N \epsilon} .
$$

Although there is no rigorous criteria for defining a maximally entangled multipartite state, there are various reasons for describing the $N$-partite $|\mathrm{GHZ}\rangle$ states as maximally entangled [20]. For instance, the $N$-qubits $|\mathrm{GHZ}\rangle$ states exhibit the maximum violations of multiparty inequalities imposed by local realistic theories (see [20] and references therein). To have an idea of how much entanglement the GHZ state contains, note that the marginal density matrix $\rho_{1}$ associated with any of the $N$ qubits corresponds to the totally mixed qubit state $\frac{1}{2} I_{2}$, which has the maximum possible von Neumann entropy, namely $\ln 2$ (and also the maximum possible value of the $\mathcal{E}$-measure, namely $\mathcal{E}=1 / 2$ ). This means that, when considering the $N$-qubit system as partitioned into a single qubit subsystem and an $(N-1)$-qubits subsystem, the GHZ state exhibits maximum entanglement.

Let us now consider the (energetically symmetric) $N$-qubits state

$$
|\mathrm{ESSLE}\rangle=\frac{1}{\sqrt{2}}|00 \ldots 0\rangle+\frac{1}{\sqrt{2 N}}\{|100 \ldots 0\rangle+|010 \ldots 0\rangle+\cdots+|00 \ldots 01\rangle\} .
$$

This state corresponds, for $N=2$, to the $s$-case (see equations (27)) with $s=-\infty$ and $\lambda=1 / 2$ (which, as far as the values of $\mathcal{E}$ and $\tau / \tau_{\min }$ are concerned, is equivalent to the state (33) with $s=0$ and $\lambda=1 / 2$ ). The state (42) takes a time

$$
\tau=\frac{\pi \hbar}{\epsilon}
$$


to evolve into the orthogonal state

$|\mathrm{ESSLE}\rangle^{\perp}=\frac{1}{\sqrt{2}}|00 \ldots 0\rangle-\frac{1}{\sqrt{2 N}}\{|100 \ldots 0\rangle+|010 \ldots 0\rangle+\cdots+|00 \ldots 01\rangle\}$.

The state $|\mathrm{ESSLE}\rangle^{\perp}$, in turn, takes a time $\tau$ to evolve back to the state $|\mathrm{ESSLE}\rangle$. The energy's expectation value and the energy's dispersion of the state $\mid$ ESSLE $\rangle$ are given by

$$
E=\Delta E=\frac{\epsilon}{2}
$$

and, from equation (4), it follows that this state saturates the speed limit,

$$
\tau=\tau_{\min }
$$

In order to compare the amount of entanglement exhibited by |ESSLE $\rangle$ with that associated with $|\mathrm{GHZ}\rangle$ we need an appropriate measure of $N$-qubit entanglement. The study of the properties and applications of multipartite entanglement measures has been the focus of an intense research activity in recent years [21-30]. An $N$-qubit entanglement measure for pure states $|\phi\rangle$ was advanced by Meyer and Wallach [22] that was later shown by Brennen [23] to be equivalent to the average of all the single-qubit linear entropies,

$$
Q(|\phi\rangle)=2\left(1-\sum_{k=1}^{N} \operatorname{tr} \rho_{k}^{2}\right) .
$$

Here $\rho_{k}, k=1, \ldots, N$, stands for the marginal density matrix describing the $k$ th qubit of the system after tracing out the rest. This quantity, often referred to as 'global entanglement' (GE), measures the average entanglement of each qubit of the system with the remaining $(N-1)$ qubits. The GE measure is widely regarded as a legitimate, useful and practical $N$-qubit entanglement measure [23-27]. This measure is invariant under local unitary transformations and non-increasing on average under local quantum operations and classical communication. In other words, $Q$ is an entanglement monotone. Another desirable property of this measure is that it can be observed without the need for full quantum state tomography [23]. This measure has been applied to the study of several problems involving multipartite entanglement, such as entanglement generation by nearly random operators [24] and by operators exhibiting particular matrix element distributions [25], thermal entanglement in multi-qubit Heisenberg models [26], and multipartite entanglement in one-dimensional time-dependent Ising models [27]. Generalizations of the GE entanglement measure involving the average values of the linear entropies associated with more general partitions of the $N$-qubit systems into two subsystems (and not only the partitions of the system into a 1-qubit subsystem and an $(N-1)$ subsystem) have also been recently the focus of considerable interest [28-30]. In particular, Scott [28] recently studied interesting properties of the family of multiqubit entanglement measures given by

$Q_{m}(|\phi\rangle)=\frac{2^{m}}{2^{m}-1}\left(1-\frac{m !(N-m) !}{N !} \sum_{s} \operatorname{tr} \rho_{s}^{2}\right), \quad m=1, \ldots,[N / 2]$,

where the sum runs over all the subsystems $s$ consisting of $m$ qubits, $\rho_{s}$ are the corresponding marginal density matrices, and $[x]$ denotes the integer part of $x$. The quantities $Q_{m}$ measure the average entanglement between all the subsystems consisting of $m$ qubits and the remaining $N-m$ qubits. The measures $Q_{m}$ have been applied to the study of quantum error correcting codes and to the analysis of the (multipartite) entangling power of quantum evolutions [28]. Another way of characterizing the global amount of entanglement exhibited by an $N$-qubit state is provided by the sum of the (bi-partite) entanglement measures associated with all the possible bi-partitions of the $\mathrm{N}$-qubits system [21]. For the particular families of (symmetric) states that 
we are going to consider here, the main conclusions obtained from the (1-qubit): $((N-1)$ qubits) bi-partitions are the same as those obtained when taking into account all the possible bi-partitions.

We are now going to use the $N$-qubit measures of entanglement (47) and (48) to compare the amount of entanglement exhibited by the $\mid$ ESSLE $\rangle$ and the $|G H Z\rangle$ states. Because of the symmetry of these states, the average of all the single-qubit linear entropies is equal to the linear entropy of the marginal density matrix associated with just one qubit,

$$
\rho_{1}=\operatorname{Tr}_{2, \ldots, N}\{|\mathrm{ESSLE}\rangle\langle\mathrm{ESSLE}|\},
$$

which is given by

$$
\rho_{1}=\left(\frac{1}{2}+\frac{N-1}{2 N}\right)|0\rangle\left\langle 0\left|+\frac{1}{2 \sqrt{N}}\{|0\rangle\langle 1|+| 1\rangle\langle 0|\}+\frac{1}{2 N}\right| 1\right\rangle\langle 1| .
$$

The eigenvalues of $\rho_{1}$ are

$$
\lambda=\frac{1}{2}\left\{1 \pm \sqrt{1-\frac{N-1}{N^{2}}}\right\}
$$

which, in the limit of a large number of qubits, yield

$$
\lim _{N \rightarrow \infty} \lambda=0,1 \text {. }
$$

The GE entanglement measure of the ESSLE state is given by the single-qubit linear entropy,

$$
Q(|\mathrm{ESSLE}\rangle)=2\left[1-\operatorname{Tr}\left(\rho_{1}^{2}\right)\right]=\frac{N-1}{N^{2}} .
$$

It is important to stress that this single-qubit marginal entropy actually represents a global property of the complete $N$-qubits system: the entropy $1-\operatorname{Tr}\left(\rho_{1}^{2}\right)$ measures the amount of entanglement between each of the $N$ qubits and the remaining $N-1$ qubits. We see that $Q(|\mathrm{ESSLE}\rangle)$ is a decreasing function of $N$, and tends to 0 when $N \rightarrow \infty$. Consequently, the amount of entanglement between each qubit of the system and the remaining $(N-1)$ qubits tends to zero as $N$ increases. Therefore, as $N$ increases, the amount of entanglement exhibited by $\mid$ ESSLE $\rangle$, as measured by the $N$-qubit GE measure, becomes much smaller than the amount of entanglement associated with $|\mathrm{GHZ}\rangle$. However, the state $\mid$ ESSLE $\rangle$ does saturate the quantum speed limit. We thus see that, as the number of qubits of the system increases, only a small amount of entanglement (as compared with the entanglement exhibited by the $|\mathrm{GHZ}\rangle$ state) is needed to obtain an energetically symmetric state that saturates the quantum speed limit.

It is also useful to evaluate upon the state $\mid$ ESSLE $\rangle$ the more general $N$-qubit entanglement measure $Q_{m}$ (given by equation (48)),

$$
Q_{m}(|\mathrm{ESSLE}\rangle)=\frac{2^{m}}{2^{m}-1}\left[\frac{m(N-m)}{2 N^{2}}\right] .
$$

We see that, for any given $m$, the measure $Q_{m}(|\mathrm{ESSLE}\rangle)$ goes to zero as $N \rightarrow \infty$. For even values of $N$, we can also consider the case $m=N / 2$ (that is, considering the average entanglement associated with all partitions of the system into two subsystems with $N / 2$ qubits each). In that case we obtain, for $N \rightarrow \infty$,

$$
Q_{N / 2}(|\mathrm{ESSLE}\rangle) \rightarrow \frac{1}{8}
$$

which is again much smaller than the value $1 / 2$ associated with the $|G H Z\rangle$ state.

Consider now the states

$$
|N ; M\rangle=\frac{1}{\sqrt{2}}|00 \ldots 0\rangle+\sqrt{\frac{M !(N-M) !}{2 N !}}\{|1 \ldots 10 \ldots 0\rangle+\ldots+|0 \ldots 01 \ldots 1\rangle\},
$$


where

$$
1 \leqslant M \leqslant N
$$

The sum within curly brackets in (56) consists of all the $\left(\begin{array}{l}N \\ M\end{array}\right)$ factorizable $N$-qubits states with $M$ qubits in state $|1\rangle$ and $(N-M)$ qubits in state $|0\rangle$. The case $M=1$ corresponds to the previously defined $|\mathrm{ESSLE}\rangle$ state, while in the case $N=M$ the $|\mathrm{GHZ}\rangle$ state is recovered. All the states $|N ; M\rangle$ are energetically symmetric, and all of them evolve to an orthogonal state in the shortest possible time (that is, all of them saturate the speed bound). The time required by $|N ; M\rangle$ to reach an orthogonal state is

$$
\tau(N ; M)=\frac{\pi \hbar}{M \epsilon} .
$$

For a given value of $N$, the amount of entanglement associated with $|N ; M\rangle$ increases with $M$, adopting its maximum value for the $|\mathrm{GHZ}\rangle$ state. We thus see that for the family of states $|N ; M\rangle$ there is a correlation between the absolute time required to reach an orthogonal state and the amount of entanglement. Within this family of states, the states with higher entanglement reach an orthogonal state sooner.

\section{Conclusions}

We have investigated some aspects of the relationship between entanglement and the speed of quantum evolution in multi-qubit systems. As was pointed out by Giovannetti, Lloyd and Maccone, both entanglement, on the one hand, and the uniformity of the distribution of energy resources among the subsystems, on the other hand, play an important role in connection with the speed of quantum evolution of multi-partite systems. Energetically symmetric, separable pure states do not saturate the speed bound. On the contrary, the speed limit can be reached for energetically symmetric, entangled states. However, how much entanglement is needed to reach the aforementioned bound? In the case of two-qubits, we found that maximally entangled states are not needed for that purpose. There are energetically symmetric states of (relatively) low entanglement that saturate the bound. In the present effort we have provided a systematic study of the connection between speed of evolution and entanglement for twoqubits pure states (summarized in figure 1) paying special attention to the role played by the distribution of the energy resources among the qubits. In particular, we showed that there is a triangular-shaped, physically forbidden region in the $\left(\mathcal{E}-\tau / \tau_{\min }\right)$-plane, involving states of low entanglement saturating the speed limit. On the line $\tau_{\min }=\tau$, corresponding to the maximum quantum speed, this region gives the entanglement gap $1 / 4<\mathcal{E}<1$, corresponding to entanglement values that can not be realized by states saturating the quantum speed limit.

We have also constructed energetically symmetric states of low entanglement (ESSLE) for $N$-qubits that evolve at the speed limit. The ESSLE states become less and less entangled (in comparison with the GHZ state) as $N$ increases. Thus, we can conclude that for large $N$ very little entanglement is needed for an energetically symmetric state to reach the quantum speed limit. In the present effort we have only discussed pure states. It would be important to extend some of the present considerations to mixed states of multi-qubit systems. In that case, it would be interesting to explore systematically the correlations existing between entanglement and the time needed to reach a state with a given fidelity distance with respect to the initial state, in connection with the role played here by the evenness of the distribution of energy resources among the subsystems. The case of mixed states, however, is considerably more complicated than the case of pure states [14] and, consequently, it seems that the only way to conduct such a study is by recourse to a systematic numerical survey of the state-space. 


\section{Acknowledgments}

The financial assistance of the National Research Foundation (NRF; South African Agency) towards this research is hereby acknowledged. Opinions expressed and conclusions arrived at, are those of the authors and are not necessarily to be attributed to the NRF. MC acknowledges support from the MEC grant FIS2005-02796 (Spain) and from the Government of the Balearic Islands. A word of thanks to Johan J v Rensburg for helpful discussions.

\section{References}

[1] Bengtsson I and Zyczkowski 2006 Geometry of Quantum States: An Introduction to Quantum Entanglement (Cambridge: Cambridge University Press)

[2] Nielsen M A and Chuang I L 2000 Quantum Computation and Quantum Information (Cambridge: Cambridge University Press)

[3] Lo H-K, Popescu S and Spiller T (ed) 1998 Introduction to Quantum Computation and Information (River Edge: World Scientific)

[4] Bouwmeester D, Ekert A and Zeilinger A (ed) 1998 The Physics of Quantum Information (Berlin: Springer)

[5] Bennett C H, Brassard G, Crepeau C, Jozsa R, Peres A and Wootters W K 1993 Phys. Rev. Lett. 70 1895-9

[6] Bennett C H and Wiesner S J 1993 Phys. Rev. Lett. 69 2881-4

[7] Ekert A and Jozsa R 1996 Rev. Mod. Phys. 68 733-53

[8] Giovannetti V, Lloyd S and Maccone L 2006 Phys. Rev. Lett. 96010401

[9] Galindo A and Martin-Delgado M A 2002 Rev. Mod. Phys. 74 347-423

[10] Gemmer J, Michel M and Mahler G 2004 Quantum Thermodynamics (Berlin: Srpinger)

[11] Giovannetti V, Lloyd S and Maccone L 2003 Europhys. Lett. 62 615-21

[12] Giovannetti V, Lloyd S and Maccone L 2003 Phys. Rev. A 67052109

[13] Batle J, Casas M, Plastino A and Plastino A R 2005 Phys. Rev. A 72032337

Batle J, Casas M, Plastino A and Plastino A R 2005 Phys. Rev. A 71024301

[14] Borras A, Casas M, Plastino A R and Plastino A 2006 Phys. Rev. A 74022326

[15] Rigo A, Plastino A R, Plastino A and Casas M 2000 Phys. Lett. A 2701

[16] Batle J, Casas M, Plastino A R and Plastino A 2002 Phys. Lett. A 296251

[17] Zyczkowski K, Horodecki P, Sanpera A and Lewenstein M 1998 Phys. Rev. A 58883

[18] Zanardi P, Zalka C and Faoro L 2000 Phys. Rev. A 62030301

[19] McHugh D, Ziman M and Buzek V 2006 Phys. Rev. A 74042303

[20] Braunstein L S and van Loock P 2005 Rev. Mod. Phys. 77513

[21] Brown I D K, Stepney S, Sudbery A and Braunstein S L 2005 J. Phys. A: Math. Gen. 381119

[22] Meyer D A and Wallach N R 2002 J. Math. Phys. 434273

[23] Brennen G K 2003 Quantum Inf. Comput. 3619

[24] Weinstein Y S and Hellberg C S 2005 Phys. Rev. Lett. 95030501

[25] Weinstein Y S and Hellberg C S 2005 Phys. Rev. A 72022331

[26] Cao M and Zhu S 2005 Phys. Rev. A 71034311

[27] Lakshminarayan A and Subrahmanyam V 2005 Phys. Rev. A 71062334

[28] Scott A J 2004 Phys. Rev. A 69052330

[29] Carvalho A R R, Mintert F and Buchleitner A 2004 Phys. Rev. Lett. 93230501

[30] Aolita M and Mintert F 2006 Phys. Rev. Lett. 97050501

[31] Margolus N and Levitin L B 1998 Physica D 120188

[32] Caves C M and Drummond P D 1994 Rev. Mod. Phys. 66481

[33] Lloyd S 2000 Nature 4061047

[34] Lloyd S 2002 Phys. Rev. Lett. 88237901 